\title{
Prekarität und Macht. Disziplinierung im System der Auswahlprüfungen
}

Prekarität gehört inzwischen „zu den großen Themen der sozialwissenschaftlichen Gegenwartsanalyse und Zeitdiagnostik“ (Aulenbacher). In der aktuellen Diskussion wird die Machtdimension unsicherer Arbeits- und Lebensverhältnisse jedoch häufig vernachlässigt. Der Beitrag analysiert die Disziplinierungsmechanismen diskriminierender Prekarität und fragt nach Ansatzpunkten für eine erneuerte Sozialkritik.

\section{Einleitung}

Die disziplinierende Wirkung von Prekarität beobachteten wir als Forschergruppe erstmals beim Einsatz ostdeutscher Leiharbeiter in der westdeutschen Autoindustrie (vgl. Brinkmann et al. 2006). Ursprünglich in einem Containerdorf untergebracht, hatten sich die Leiharbeiter organisiert, mit Unterstützung der IG Metall eine Tarifkommission gewählt und eine bessere Entlohnung sowie günstigere Arbeits- und Wohnbedingungen durchgesetzt. Trotz Bereitschaft zu kollektiver Aktion waren die Leiharbeiter jedoch bestrebt, die ihnen zugewiesenen Arbeitsaufgaben in hoher Qualität und zur größten Zufriedenheit von Kollegen und Vorgesetzten zu erfüllen. Es handelte sich um fügsame Arbeitskräfte, die insgeheim vom Sprung in die Festanstellung träumten. Von ihrer Bereitschaft zur Selbstdisziplinierung ging zugleich eine subtile Wirkung auf Stammbeschäftigte aus, die wir als „Bumerangeffekt“ (Dörre 2009, S. 58) bezeichneten.

Inzwischen können wir diese Beobachtung verallgemeinern. Prekarisierungsprozesse, so die hier vertretene These, konstituieren ein Disziplinarregime. Dieses Regime beruht auf Auswahlprüfungen. Eine Quasi-Institutionalisierung von Prüfungsformaten, für die sich prekär Beschäftigte in der Hoffnung auf ein besseres Leben qualifizieren müssen, verstärkt ein Machtgefälle am Arbeitsmarkt, das allmählich den gesamten Lebenszusammenhang strukturiert. Auswahlprüfungen vermitteln jenen, die in Unsicherheit leben müssen, eine besondere Selbstwahrnehmung. Den Prekarisierten wird ein Sonderstatus zugeschrieben, den sie selbst als
Minderheitenperspektive erleben. Um die darin angelegten Machtasymmetrien analytisch erfassen zu können, empfiehlt es sich, diesen Sonderstatus genauer in den Blick zu nehmen.

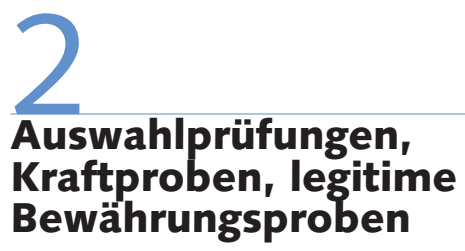

Ein solches Vorhaben kann an die pragmatische Soziologie Luc Boltanskis anknüpfen. Deren Grundgedanke lautet, dass sich die Akzeptanz sozialer Ungleichheit und Unsicherheit durch eigensinniges Handeln von Akteuren in obligatorischen Auswahlprüfungen erklären lässt. Für diese Prüfungen müssen sich Menschen qualifizieren, um Zugang zu bestimmten sozialen Positionen zu erhalten. Elementar für den Ansatz sind die Begriffe Kraftprobe und Bewährungsprobe. Eine Gesellschaft kann „durch die Natur der von ihr begründeten Bewährungsproben definiert werden“" (Boltanski/Chiapello 2003, S. 74). Gesellschaften konfrontieren Individuen (Mikroperspektive), aber auch Klassen von Individuen (Makroperspektive) immer wieder mit Situationen, in denen sie ihre Kräfte messen, eben mit Bewährungsproben.

Als bloße Kraftproben münden Bewährungsproben in eine Feststellung und gegebenenfalls in eine Fixierung gesellschaftlicher Kräfteverhältnisse. Moralische Urteile spielen dabei keine Rolle; es geht allein um den Einsatz von Machtressourcen, um Sachverhalte ohne Werturteil. Anders verhält es sich in der Dimension sozialer Ordnungen, wo das Kräftemessen einem Rechtfertigungszwang unterliegt. In legitimen Bewährungsproben wird über die Wertigkeit von Personen und Personengruppen im sozialen Gefüge geurteilt. Legitime Bewährungsproben kommen daher nicht ohne Gerechtigkeitsvorstellungen aus.

Der Übergang von der Kraftprobe zur legitimen Bewährungsprobe beinhaltet „soziale Identifizierungs- und Qualifizierungsbemühungen“, in welchen die sozialen Akteure ihre Ressourcen offenlegen und unterscheidbar machen. Denn um von einem Gerechtigkeitsstandpunkt aus überhaupt bewertbar zu sein, benötigen Bewährungsproben ein eindeutig bestimmbares Format. Es muss sich um besondere Situationen mit Prüfungscharakter handeln - sei es nun ein Wettrennen, eine Lateinklausur oder auch die Qualifizierung für eine Festanstellung. Zur Anwendung kommen nur jene Ressourcen, die dem Charakter der Auswahlprüfung entsprechen. Es obliegt den Institutionen, Bewährungsproben „eine Form zu geben, ihren Ablauf zu kontrollieren und so dem illegitimen Einsatz externer Ressourcen vorzubeugen“, um Gerechtigkeit zu wahren (ebd., S. 73). In Gesellschaften, in denen viele Bewährungsproben Rechtfertigungszwängen unterliegen, „wird die Stärke der Starken gemindert" (ebd.). Dennoch handelt es sich bei Kraftproben und legitimen Bewährungsproben nicht um einander ausschließende Mechanismen. In Auswahlprüfungen sind stets beide Dimensionen aufeinander bezogen.

Klaus Dörre, Prof. Dr., lehrt am Institut für Soziologie der Universität Jena. Arbeitsschwerpunkte: Arbeits-, Industrie- und Wirtschaftssoziologie. e-mail: klaus.doerre@uni-jena.de 


\section{Das Disziplinarregime der Prekarisierung}

Wie lässt sich diese abstrakte Überlegung auf Prekarisierungsphänomene beziehen? Unsere Analyse nutzt die Kategorie der Auswahlprüfungen ohne Boltanskis Konzeption in Gänze zu übernehmen. Zeitgenössische Formen von Prekarität resultieren wesentlich aus dem Bemühen von Unternehmen, widersprüchlichen Interessen und Handlungsanforderungen zu entsprechen. Angesichts der Anforderungen schwankender Märkte laufen solche Bemühungen im Grunde auf eine Quadratur des Kreises hinaus. Einerseits gibt es ein Interesse exportorientierter Unternehmen, loyale, qualifizierte Stammbeschäftigte zu binden; andererseits sollen Renditen stabil gehalten, Anforderung von Analysten und Eigentümern bedient und gegebenenfalls auch Entlassungskosten vermieden werden. Solche Widersprüchlichkeit sucht das Management seit jeher mittels Konstruktion von Sondergruppen zu meistern. Ethnische und Geschlechterkonstruktionen werden genutzt, um Frauen oder Migranten als Zuverdienerinnen oder auch als Arbeitskräfte mit „Gaststatus“ in die weniger attraktiven Segmente betrieblicher Arbeitsmärkte zu lenken und ihnen zugleich besondere Flexibilisierungsleistungen abzuverlangen (Balibar/Wallerstein 1988).

Prägend für das zeitgenössische Disziplinarregime ist jedoch nicht die Instrumentalisierung von Ethnie und Genus, neu ist vielmehr, dass es einen zuvor unbekannten Sonderstatus diskriminierender Prekarität erzeugt. Dies geschieht mittels einer QuasiInstitutionalisierung von Auswahlprüfungen, die auf unterschiedlichen Stufen der sozialen Hierarchie Zugänge nicht nur zu auskömmlichen Löhnen, halbwegs sicherer Beschäftigung und akzeptablen Lebensbedingungen, sondern auch zur Verfügung über Sozialeigentum (Castel 2005, S. 42f.) und zu sozialen Netzen regulieren. Die Quasi-Institutionalisierung von Prüfungen erfolgte im Zuge einer marktgetriebenen Landnahme, in deren Verlauf die zuvor enge Koppelung von Lohnarbeit mit sozialen Schutz- und Partizipationsrechten allmählich aufgebrochen wurde. Die Verteilung von knappem Sozialeigentum verläuft nunmehr über - häufig geschlechterdiskriminierende - Prüfungsformate, die sich in und neben den regulären beruflichen Integrationsformen etabliert haben. Flexibilisierungsleistungen, die prekär Beschäftigte und Ausgegrenzte in diesen Bewährungsproben erbringen müssen, werden in der Regel nicht zertifiziert, d.h. sie erfolgen häufig informell. Angeregt durch empirische Forschungen lassen sich drei Prüfungsformate unterscheiden: die betrieblichen Auswahlprüfungen an der Schnittstelle von Stammbelegschaft und flexibel Beschäftigten (Abschnitt 3.1), die überwiegend von staatlichen Akteuren initiierten Auswahlprüfungen an der Schnittstelle von Beschäftigungssystem und Erwerbslosigkeit (Abschnitt 3.2) sowie die Auswahlprüfungen des Lebenslaufregimes an der Schnittstelle von Erwerbsarbeit und Reproduktionstätigkeit (Abschnitt 3.3).

\subsection{BETRIEBLICHE AUSWAHL- PRÜFUNGEN}

\section{CHARAKTERISTIKA}

Betriebliche Auswahlprüfungen ${ }^{1}$ regulieren die Machtbeziehungen zwischen Stammbelegschaft und flexibel Beschäftigten. Im Falle einer „notwendigen Prekarität" in Arbeitsmärkten mit austauschbaren Qualifikationen funktioniert das Ausleseprinzip allerdings anders als bei einer „transitorischen Prekarität“ in berufsfachlichen oder der „avantgardistischen Prekarität" in Künstlerarbeitsmärkten (Pelizzari 2009). In sämtlichen Teilarbeitsmärkten wirkt das Rechtfertigungsregime jedoch über ein scheinbar meritokratisches Aufstiegsversprechen. Flexibler Beschäftigung wird im gesellschaftlichen Rechtfertigungsregime eine arbeitsmarktpolitische Integrationsfunktion, ein Klebe- oder Brückeneffekt zugerechnet. Wer gut arbeitet und sich bewährt, soll die Chance zu einem Sprung in bessere Beschäftigung haben. Die Entscheidungsbefugnisse liegen primär beim Management. Häufig sind jedoch Mitglieder von Stammbelegschaften oder deren organisierte Repräsentanten an der Definition von Prüfungssituationen beteiligt. Allerdings weichen die Prüfungsformate vom Eindeutigkeitsgebot ab, das legitime Bewährungsproben auszeichnet. Sie haben überwiegend etwas Unbestimmtes, Diffuses, Unkalkulierbares. Ob und zu welchen Bedingungen ein Leiharbeiter oder eine Aushilfskassiererin den Sprung in die Stammbelegschaft schafft, ist in den meisten Fällen ungeklärt. Auch die Res- sourcen, die eingebracht werden müssen, sind keineswegs genau spezifiziert. Die fachliche Qualifikation ist in solchen Auswahlprüfungen nur eine Mindestbedingung. Erwartet werden außergewöhnliche Flexibilität, überdurchschnittliches Engagement und die Fähigkeit, sich rasch auf veränderte Tätigkeiten, neue Kollegen und Vorgesetzte einstellen zu können - und dies alles zu häufig deutlich schlechteren Konditionen, als sie Stammbeschäftigten geboten werden. In solch prekären Anstellungsverhältnissen wird generell „auf Probe“ gearbeitet. Sicher gibt es formale Prüfungen, Bewerbungen, Einstellungsgespräche etc., aber die Bewährungszeit endet eigentlich nie. Gleich ob Leiharbeit, Befristungen oder Werkverträge - immer seltener werden entsprechende Verträge aufgrund eines tatsächlich zeitlich befristeten Arbeitsanfalls abgeschlossen. Bei den Nutzungsmotiven der Beschäftiger treten stattdessen „Risikoexternalisierung“ und „Personalerprobung" in den Vordergrund (Hohendanner 2010; Holst et al. 2009).

\section{DISZIPLINIERUNGSMECHANISMEN}

Die betrieblichen Prüfungsformate beinhalten eine Vielzahl an Disziplinierungsmechanismen. Dazu gehört zunächst die Selbstdisziplinierung prekär Beschäftigter, die sich für eine Festanstellung oder auch nur für eine Verlängerung ihres Arbeitseinsatzes empfehlen wollen. Gerade weil die Aussichten auf eine Festanstellung gering sind, gleichen die betrieblichen Bewährungsproben einem permanenten Wettrennen. Es geht zu wie im Leistungssport. Von vornherein ist klar, dass nur wenige das Ziel einer Übernahme in die Stammbelegschaft erreichen können. Folglich müssen alle verfügbaren individuellen Ressourcen mobilisiert werden, um den Sprung zu schaffen. Das Ziel ist jedoch keine Spitzenleistung, sondern Anschluss

\footnotetext{
Nachfolgende Ausführungen beruhen auf den Projekten „Funktionswandel von Leiharbeit" (12 betriebliche Fallstudien, 72 Interviews mit Stammbeschäftigten, Leiharbeitern, Vorgesetzten und Interessenvertretungen: Holst et al. 2009); „Flexibilität und Stabilität im Wertschöpfungssystem Automobil“ (standardisierte Befragung von Beschäftigten eines Automobilwerks, $n=1442$ plus qualitative Befragung betrieblicher Experten, $\mathrm{n}=55$ : Holst/Matuschek 2011; Dörre et al. 2011 sowie eine Studie in fünf Betrieben der optischen Industrie (standardisierte Belegschaftsbefragung $\mathrm{n}=459$; qualitative Studie, $\mathrm{n}=14$ : Behr 2009; Dörre et al. 2009).
} 
an „Normalität“, an Standards, die noch immer von den Stammbeschäftigten und vor allem von den verantwortlichen Vorgesetzten definiert werden. Auf diese Weise gelingt es in betrieblichen Auswahlprüfungen, Hoffnungen unsicher Beschäftigter leistungspolitisch zu instrumentalisieren, obwohl immer klar ist, dass die Versuche zur „Normalisierung“ der beruflichen Biografie höchst selten von Erfolg gekrönt sind.

Die Anpassungsbereitschaft der Prekären wird durch den Disziplinierungsdruck, der von Festangestellten ausgeht, noch verstärkt. Prekär Beschäftigte müssen bei jedem Einsatz, bei jedem Arbeitsplatz- oder Betriebswechsel aufs Neue um die Anerkennung ihrer Kollegen und Vorgesetzten ringen. Die beteiligten Festangestellten entscheiden mit darüber, ob und wem der Sprung in sichere Beschäftigung gelingt. Insofern sind Mitglieder von Stammbelegschaften stets mehr oder minder aktiv an Auswahlprüfungen beteiligt. Die Prüfungssituation ist für die Prekären auf Dauer gestellt, und deshalb üben die beteiligten Festangestellten zumindest indirekt Druck auf die unsicher beschäftigten „Minderheiten“ aus. Teilweise unbeabsichtigt, betätigen sich Kolleginnen und Kollegen als Teil des Selektionssystems. Eine solche Klasse „uneindeutiger" Auswahlprüfungen fördert zugleich eine exklusive Solidarität von Stammbelegschaften. Es kann durchaus im Interesse gesicherter Beschäftigtengruppen liegen, Konkurrenz durch annähernde Gleichstellung prekär Arbeitender abzumildern. Spätestens wenn die eigene Beschäftigungssicherheit bedroht ist, sind die Grenzen solidarischen Verhaltens jedoch oftmals erreicht. ${ }^{2}$

Schließungstendenzen in Stammbelegschaften werden zusätzlich durch Disziplinierungseffekte gefördert, die von unsicher Beschäftigten auf Festangestellte ausstrahlen. Dergleichen zu behaupten, mag auf den ersten Blick gewagt erscheinen, denn prekär Beschäftigte wirken häufig geradezu ohnmächtig. Und doch sind sie in der Lage, Strategien in der Konkurrenz zu entwickeln, mit denen sie Stammbelegschaften unter Druck setzen. Je bereitwilliger sich unsicher Beschäftigte den betrieblichen Auswahlprüfungen unterziehen und je klagloser sie die prekären Arbeitsbedingungen akzeptieren, desto unkalkulierbarer wird ihre Präsenz für die Stammbeschäftigten. Durch ange- passtes Verhalten erzeugen die Prekären eine „Ungewissheitszone“ (Crozier/Friedberg 1993), die für Festangestellte kaum zu kontrollieren ist. Die Herstellung von Ungewissheit ist der Part, den prekarisierte Gruppen in betrieblichen Machtspielen ausüben. Teilweise beruht diese „Macht in der Ohnmacht" auf unmittelbarer Konkurrenz, etwa wenn eine mit Leiharbeitern besetzte Schicht immer wieder die Akkordleistung der Festangestelltenschicht übertrifft und so den informellen Konsens zur Leistungsregulation sprengt. Der Disziplinierungseffekt findet sich jedoch auch bei betrieblichen Statusgruppen, die von der unmittelbaren Konkurrenz gar nicht betroffen sind. Ihnen wird immer wieder vor Augen geführt, wie mit betrieblichen „Minderheiten“ umgesprungen werden kann, die trotz aller Gefügigkeit stets zu den ersten gehören, wenn Entlassungen auszusprechen oder unangenehme Jobs zu verteilen sind.

Dies vor Augen, muss die Festanstellung geradezu als Privileg erscheinen. Um ihre Position zu verteidigen, tendieren auch Stammbeschäftigte zur Selbstdisziplinierung. Ihre Bereitschaft, sich ständiger Auswahlprüfungen auszusetzen, wächst infolge des Anschauungsunterrichts, den prekarisierte Gruppen unfreiwillig leisten. Gerade in Krisenzeiten müssen sich auch Festangestellte mittels besonderer Flexibilisierungsleistungen stets aufs Neue für ihr unbefristetes Arbeitsverhältnis qualifizieren. Sei es, indem sie Kurzarbeit und Lohnabschläge in Kauf nehmen, sei es, indem sie sich in andere Werke ihres Konzerns ausleihen lassen. Auch die Festanstellung ist somit immer nur eine unter Vorbehalt. Zwar ist die Kündigung unwahrscheinlich, aber weder die Tätigkeit noch der Einsatzort, die Abteilung, die Arbeitszeit oder die Entlohnung sind dauerhaft im Sinne eines erwünschten Optimums gesichert.

\subsection{AUSWAHLPRÜFUNGEN DES ARBEITSMARKTREGIMES}

Im Wechselspiel von Fremd- und Selbstdisziplinierung betrieblicher Statusgruppen wird die Funktionsweise des mit Prekarisierungsprozessen verwobenen Disziplinarregimes sichtbar. In ihren Gruppenkonkurrenzen befangen, machen sich Prekäre wie Festangestellte zu gefügigen Arbeitskräften. Sie spüren die Macht der jeweils benachbarten Statusgruppen. Ei- gentümer und strategiefähige Manager, die mittels Renditevorgaben und Zielvereinbarungen herrschen, geraten tendenziell aus dem Blick. Spannungen entstehen bevorzugt zwischen subalternen Statusgruppen, die um den Erhalt oder die Verbesserung ihrer sozialen Positionen kämpfen. Wer permanent in solche Kämpfe verstrickt ist, wird unduldsam und neigt zu exklusiver Solidarität. Leiharbeiter gelten einer Mehrheit der Stammbeschäftigten als "Arbeitnehmer zweiter Klasse“ (Holst/ Matuschek 2011), wie andere prekär Beschäftigte werden sie jedoch als bemühte Arbeitskräfte respektiert. Das ist im Falle von Langzeitarbeitslosen anders. Obwohl eine Mehrheit „Hartz IV“ kritisch bewertet, ist mehr als die Hälfte der befragten westdeutschen Arbeiter und Angestellten der Meinung, auf Arbeitslose solle größerer Druck ausgeübt werden (54\%). Nahezu $50 \%$ halten die Aussage „Eine Gesellschaft, in der jedermann aufgefangen wird, ist nicht überlebensfähig " für zutreffend. ${ }^{3}$ In solchen Formen einer ausschließenden Solidarität deutet sich bereits die Wirkung einer besonderen Klasse von Auswahlprüfungen an, mit deren Hilfe Aktivitäten an der Schwelle von Erwerbstätigkeit und Arbeitslosigkeit reguliert werden. ${ }^{4}$ In den Bewährungsproben des aktivierenden Arbeitsmarktregimes entscheidet sich aus einer „Mehrheitsperspektive“, ob eine Person weiterhin respektiertes Mitglied der Gesellschaft ist, oder ob sie sich in einem wenig anerkannten Status der Fürsorge einrichten muss.

2 Dementsprechend ambivalent ist die Haltung vieler Festangestellter gegenüber den Leiharbeitern. Bei $41,8 \%$ der von uns befragten Beschäftigten eines Automobilwerks stößt die Aussage "Leiharbeit wird auch genutzt, um Konkurrenz in die Betriebe zu bringen" auf Zustimmung (31 \% Ablehnung), und ein noch größerer Anteil (42,9\%) bestreitet, dass Leiharbeiterinnen "genauso zur Betriebsfamilie“ gehören wie Stammkräfte (Holst/Matuschek 2011).

3 Im Osten stoßen solche Statements überwiegend auf Ablehnung, vgl. Dörre et al. 2011, 2009.

4 Soweit nicht anders ausgewiesen, beruhen die nachfolgenden Ausführungen auf Ergebnissen unseres von der Deutschen Forschungsgemeinschaft (DFG) geförderten Forschungsvorhabens

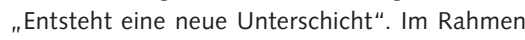
der qualitativen Erhebung wurden bislang Leistungsbezieher des ALG II ( $11=99, \mathrm{n} 2=70)$ sowie Expertinnen und Experten aus Arbeitsverwaltungen, Trägern, Selbsthilfegruppen und Verbänden $(\mathrm{n} 1=53, \mathrm{n} 2=20)$ in zwei Wellen befragt (Booth et al. 2011). 


\section{FORMATE UND AKTEURE}

Die Prüfungsformate an dieser Nahtstelle werden hauptsächlich von staatlichen Akteuren festgelegt. Neben dem Gesetzgeber spielen die Arbeitsverwaltungen bei der Definition von Fördermaßnahmen ebenso wie bei der Durchsetzung strenger Zumutbarkeitsregeln eine entscheidende Rolle. Auf der regionalen Ebene beeinflussen die politischen Entscheider mit arbeitspolitischen Programmen oder der Festlegung sogenannter Problemgruppen zugleich die Prüfungsformate. Die Spitzen von AR$\mathrm{GEN}^{5}$ modifizieren Regelungen und prägen so besondere regionale Regimes. Auf der Sachbearbeiterebene werden Leistungsansprüche geprüft und Fördermaßnahmen oder Arbeitsgelegenheiten zugeteilt.

Der gesamte Prozess der Durchsetzung von Instrumenten und Verfahrensregeln einer aktivierenden Arbeitsmarktpolitik kann als Etablierung neuer Prüfungsformate interpretiert werden. Dass die implementierten Auswahlprüfungen einen disziplinierenden Charakter besitzen, entspricht den Intentionen einer Arbeitsmarktpolitik, die Förderung bietet, welche sich Arbeitslose mittels Eigenaktivitäten erst verdienen müssen. Als Quasi-Unternehmer ihres eigenen Arbeitsvermögens sollen sich die Leistungsbezieher mehr oder minder eigenverantwortlich in die Lage versetzen, reguläre Arbeit zu finden oder zumindest ihre Situation zu verbessern. Um den Anreiz für Eigenaktivitäten zu erhöhen, soll der Status des oder der Langzeitarbeitslosen bewusst „ungemütlich “ gehalten werden. Schon weil in dieser Ausrichtung eine Passivitätsvermutung mitschwingt, wird die über Auswahlprüfungen vollzogene Statuszuweisung seitens der Arbeitslosen überwiegend als Stigmatisierung wahrgenommen. "Als Hartzi bist Du nichts“, lautet eine bezeichnende Einschätzung, mit der wir so oder ähnlich immer wieder konfrontiert wurden.

Tatsächlich beinhaltet das Regime strenger Zumutbarkeit eine Vielzahl an Auswahlprüfungen, die Leistungsbezieher als bürokratische Disziplinierung erleben. Die Erfahrung von Fremdbestimmung beginnt mit der Einteilung in unterschiedliche Kundengruppen, die seitens der Sachbearbeiter vorgenommen wird, und sie reicht bis zu jenen Mechanismen, die eine engmaschige bürokratische Kontrolle des Alltags von Leistungsbeziehern ermöglichen. Das Regime strenger
Zumutbarkeitsregeln beschränkt sich durchaus nicht auf Sanktionen, von der nur eine Minderheit der Arbeitslosen unmittelbar betroffen ist. Es speist sich aus materieller Knappheit (Regelleistungen) und einer teilweise rigiden Kontrolle von Eigenaktivitäten (Bewerbungen, Maßnahmen, Bereitschaft zu sozial geförderter Beschäftigung). Die Strenge resultiert aus Regeln und Eingriffen, die die Lebensformen der Anspruchsberechtigten betreffen - Aufwendungsgrenzen für die Wohnung oder die Überwachung von Besitz und Zuwendungen (z.B. Höchstgrenzen für geldwerte Geschenke). Und sie resultiert auch aus ständig wiederkehrenden Missachtungserfahrungen in der gesellschaftlichen Öffentlichkeit (Booth et al. 2010).

Ein Merkmal der Auswahlprüfungen, die sowohl die individuelle Positionierung innerhalb dieses Regimes als auch die Übergänge in das System der Erwerbsarbeit regulieren, ist auch hier ihre Uneindeutigkeit. Das gilt insbesondere für die normative Dimension, für die Gerechtigkeitsmaßstäbe. Wie schon bei den betrieblichen Prüfungen beobachtet, können Bewährungsproben legal sein; Prüfern wie zu Prüfenden fehlt es jedoch an gemeinsam geteilten Gerechtigkeitsvorstellungen. Die Leistungsbezieher sind bestrebt, sich aktiv für Prüfungsformate zu qualifizieren. Reguläre Erwerbsarbeit gilt ihnen überwiegend als Norm, die nicht infrage gestellt werden darf (Bescherer et al. 2008, S. 10ff.). Ihre Bemühungen zielen jedoch teilweise auf eine Qualifizierung für Auswahlprüfungen, die oberhalb der Respektabilitätsschwelle und damit außerhalb des Regimes strenger Zumutbarkeit angesiedelt sind. Anders gesagt, die Leistungsbezieher müssen sich den Bewährungsproben des neuen Arbeitsmarktregimes unterziehen, für sinnvoll und gerecht halten sie dies jedoch häufig nicht. Insofern handelt es sich bei den Prüfungen immer auch um Kraftproben, in der die Leistungsbezieher überwiegend als atomisierte, gleichwohl eigensinnige Individuen handeln müssen.

\section{WIRKUNGSMECHANISMEN}

Um diese Kraftproben einigermaßen bestehen zu können, müssen sich die Befragten in der einen oder anderen Weise selbst disziplinieren. Eine Form der Selbstdisziplinierung ist die überschießende Aktivität. Leistungsbezieher, die wir dem Typus der „Um-jeden-Preis-Arbeitenden“ zurechnen, würden ungeachtet strenger Zumutbarkeitsregeln nahezu jede positionsverbessernde Erwerbstätigkeit akzeptieren, die ihnen ein Leben unterhalb einer „Schwelle der Respektabilität“ erspart. Maßnahmen der Arbeitsverwaltung betrachten diese Befragten häufig als überflüssig oder gar als Drangsalierung, weil das, wonach sie eigentlich streben reguläre Erwerbsarbeit - nicht im Angebot der Arbeitsagenturen ist. Die zweite Form der Selbstdisziplinierung läuft auf ein Arrangement mit widrigen Umständen hinaus. Leistungsbezieher, die dem Typus der „Als-ob-Arbeitenden“ entsprechen, wollen liebend gerne einer Erwerbstätigkeit nachgehen, müssen sich aber mehr und mehr mit „Arbeitsersatz“ abfinden. Sozial geförderte Tätigkeiten wie Ein-Euro-Jobs sind für sie keine Bestrafung, sie erlauben es, zeitweilig Fassaden der Normalität aufrecht zu erhalten. Eigenaktivitäten - sei es nun das bürgerschaftliche Engagement oder der Ein-Euro-Job - werden subjektiv so umgedeutet, als handele es sich um eine reguläre Erwerbsarbeit. Die Spannungen zwischen Erwerbsarbeitsnorm und Lebenspraxis lassen sich auf diese Weise mildern, aber niemals völlig überwinden.

Allerdings bleiben permanente Qualifizierungsversuche für betriebliche oder staatliche Auswahlprüfungen nicht ohne Folgen. Zwischen Zwang und Selbstunterwerfung ist die Disziplinierung durch Erschöpfung angesiedelt. Um ihre Situation zu verändern, müssen Arbeitslose häufig hart arbeiten. Minijob und verordnetes Praktikum können sich zeitweilig schon zu einer 48-Stunden-Woche summieren. Hinzu kommen Anforderungen aus dem Familienzusammenhang und der Kindererziehung. Trotz solcher Anstrengungen kommt ein Großteil der Befragten beruflich nicht vom Fleck. Bei einem Teil der Leistungsbezieher, unter ihnen vor allem Soloselbstständige, zeichnet sich trotz günstiger Konjunktur individuell eher eine Abwärtsmobilität ab. Lange Jahre in Arbeitslosigkeit und prekärer Beschäftigung bewirken, dass die Betroffenen regelrecht ausbrennen. Ohne eine realistische Chance auf grundlegende Besserung ihres Lebens, gehen die subjektiven Antriebskräfte für Aufstiegsbemühungen mehr und mehr verloren.

5 Die Abkürzung ARGEN steht für Arbeitsgemeinschaften nach dem Sozialgesetzbuch Zweites Buch (SGB II). 
Indem es eine reguläre Erwerbsarbeit zur einzig legitimen Zielstellung erklärt, erzeugt das Regime strenger Zumutbarkeit an der Schnittstelle von regulärer Erwerbsarbeit, sozial geförderter Beschäftigung und Erwerbslosigkeit eine soziale Hierarchie, die auf ihre Weise die Perspektive eines „Minderheitenstatus“ fixiert. Am unteren Ende dieser Hierarchie finden sich in der gesellschaftlichen Wahrnehmung die „faulen Arbeitslosen“, „Sozialschmarotzer" und „Illegalen“- Konstruktionen, von denen sich die große Mehrzahl der Leistungsbezieher abzugrenzen versucht. Real handelt es sich häufig um „Nichtarbeitende“, die die objektive Not der Erwerbslosigkeit in eine subjektive Tugend verwandeln. Darüber befinden sich in der sozialen Hierarchie je nach Situation und lebensweltlichem Kontext Ein-EuroJobber, Beinahe-Rentner, bürgerschaftlich Engagierte, Maßnahmeabsolventen oder prekäre Soloselbstständige. Trotz ihrer enormen sozialen Heterogenität zeichnet sich diese Gruppe durch eine gemeinsam geteilte Grunderfahrung aus. Sie alle fühlen sich von dem ausgeschlossen, was in der Gegenwartsgesellschaft noch immer „Normalität" garantiert. Sie alle verfügen nicht über eine dauerhafte Erwerbsarbeit, die ihnen ein akzeptables Einkommen, soziale Wertschätzung und vor allem eine halbwegs stabile Basis für eine in die Zukunft gerichtete Lebensplanung bieten könnte.

\subsection{BEWÄHRUNGSPROBEN DER LEBENSFÜHRUNG}

Dass Unsicherheitserfahrungen mehr und mehr den gesamten Lebenszusammenhang (nicht nur) prekarisierter Statusgruppen strukturieren, hängt eng mit einer Klasse von Auswahlprüfungen zusammen, die an der Schnittstelle von Öffentlichem und Privatem angesiedelt ist. Diese Auswahlprüfungen sind besonders schwer zu identifizieren, weil sie entweder indirekt wirken oder ihre Formate und Regeln nicht unmittelbar vom Staat oder den ökonomischen Eliten definiert werden. Teilweise finden sie in sozialen Netzen und damit in einer Lebenssphäre statt, die eigentlich geeignet wäre, die Folgen unsicherer Beschäftigung zumindest teilweise zu kompensieren. Entsprechende Prüfungsformate und ihre Disziplinierungsmechanismen sind noch kaum erforscht. Dennoch lässt sich sagen, dass sie eng an gesellschaftliche Zeitregimes gekoppelt sind.

\section{FEHLENDE ZEITSOUVERÄNITÄT}

In der Zeitdimension bedeutet Prekarität, den Anforderungen eines Regimes diskontinuierlicher Zeit trotz schwacher Ressourcenausstattung und großer individueller „Verwundbarkeit“ genügen zu müssen. Wiederum sind es Auswahlprüfungen im Erwerbssystem, die Flexibilitätsanforderungen mit Selektionscharakter erzeugen. Arbeitszeiten, die in der Wahrnehmung niedrig entlohnter Teilzeitbeschäftigter in Filialen eines Discounters vom Management willkürlich und vor allem kurzfristig festgelegt werden, geraten für die Beschäftigten zur permanenten Bewährungsprobe. Werkverträge, die Soloselbstständigen Fristen und Termine setzen, eignen sich nicht nur, um das Ausleseprinzip auf Dauer zu stellen, sie nehmen den Betreffenden häufig etwas von ihrer Zeitsouveränität in Beruf und Privatleben. Indem die Auftraggeber oder Vorgesetzten die Tätigkeiten, die zur Bewältigung diskontinuierlicher Arbeitszeiten und Arbeitsanforderungen notwendig sind, als Gratisressource nutzen, eignen sie sich zugleich die Verfügung über Lebenszeit und Lebenstätigkeit prekär Beschäftigter an.

In der Sphäre individueller Lebensführung wird der Kampf um ,jedes Zeitatom“ (Negt 1984, S. 27) jedoch nur selten zur offenen Kraftprobe. Dies vor allem, weil die Übersetzung von Unsicherheitserfahrungen in Maßstäbe für eine rationale Lebensführung weitgehend als Privatsache gilt. Es sind Privatpersonen, die diskontinuierliche Zeiterfahrungen, wie sie aus unstetem Einkommen und unsicherer Beschäftigung resultieren, mit den Flexibilitätsanforderungen sozialer Netze, Familienstrukturen und Reproduktionstätigkeiten in Einklang bringen müssen. Die Heterogenisierung von Lebensstilen, Haushalts- und Familienformen sowie die damit verknüpften Legitimationen geschlechtsspezifischer Arbeitsteilung erzeugen alltägliche Flexibilisierungserfordernisse, die es mit der eigenen Erwerbsarbeit oder der des Lebenspartners zu vereinbaren gilt. Hier beginnen die Auswahlprüfungen des Privaten, die im Lebenszusammenhang verankert sind. Sie erstrecken sich auf die Verteilung von Reproduktionstätigkeiten, den „Zweiten Arbeitstag“" nach der Erwerbsarbeit, den noch immer vorwiegend Frauen zu leisten haben, und sie entzünden sich an Geschlechterkonstruktionen und der Kompatibilität von Lebensstilen.
Darauf bezogene Aushandlungsprozesse, in denen stets Gerechtigkeitsvorstellungen mitschwingen, besitzen unzweifelhaft etwas Befreiendes. Je tiefer man in der sozialen Hierarchie absteigt, desto schwerer fällt es individuellen Akteuren jedoch, die Vorteile flexibler Zeit lebenspraktisch zu nutzen. In hochqualifizierten Bereichen, etwa im Wissenschaftssystem, dem Journalismus oder bei Künstlern mit ihren besonderen Formen einer transitorischen oder avantgardistischen Prekarität, mag es zumindest zeitweilig gelingen, die Nachteile unsteter Beschäftigung durch die Vorzüge von kreativer Arbeit zu kompensieren. Für einen Großteil der eigentlichen „Prekarier“ ist dergleichen schlechterdings unmöglich, weil sie sich sowohl im Erwerbssystem als auch in der Reproduktionssphäre mit einer Kumulation von Risiken und Belastungen konfrontiert sehen. Die Erschließung vielfältiger Handlungsoptionen, die das Regime diskontinuierlicher Zeit zu einer Daueraufgabe macht, wird von Männern und Frauen im prekären Sektor überwiegend als Zwang erlebt, denn unsicher Beschäftigte und selbst Arbeitslose in staatlich definierten Auswahlprüfungen leiden häufig an Zeitmangel.

Wie hochqualifizierte Freelancer sind sie permanent gefordert, jede sich bietende Chance zu nutzen, um ihre Lage zu verbessern. Zum täglichen Zwei-StundenJob im Reinigungsgewerbe gesellt sich bei einer jungen Langzeitarbeitslosen und Mutter von zwei Kindern mitunter das achtstündige Praktikum; die Teilnahme wiederum ist Voraussetzung für den Bezug von Transferleistungen. Hinzu kommen Schnäppchenjagd und Altstoffsammlung in der freien Zeit, an der sich auch die Kinder beteiligen. Der Mann, als Niedriglohnbezieher ebenfalls im Reinigungsgewerbe tätig, ist bis zum späten Abend außer Haus. In dieser familialen Konstellation wird der Alltag zu einer Dauerprüfung, deren Bewältigung eine erschöpfte Familie produziert. Übermäßiger TV-Konsum, auch schon für die Kinder, entspringt bei solchen Lebensbedingungen dem Bedürfnis, dem belastenden Alltag wenigstens zeitweilig eine Ruhephase abzugewinnen.

\section{GERINGE TEILHABECHANCEN}

Frei verfügbare Zeit wird auch und gerade im Alltagsleben prekarisierter Gruppen zu einem knappen Gut. Selbst wenn man geringfügig beschäftigt oder erwerbslos 
ist, sieht man sich in zahlreichen Bewährungsproben mit einem permanenten Aktivitätszwang konfrontiert, an dem sich Teilhabechancen entscheiden. Die Bewährungsproben wirken, obwohl wenig formalisiert und teilweise ohne Zwang zu öffentlicher Rechtfertigung, analog zum meritokratischen Prinzip, d. h. die Flexibilitätsanforderungen werden vornehmlich individuell bewältigt. Ist man selbst nicht in der Lage, die Optionenvielfalt reicher Gesellschaften für sich zu nutzen, erscheint dies subjektiv als persönliches Versagen. Die damit verbundenen Ausleseprozesse funktionieren über Ausschluss und Selbstselektion in sozialen Netzwerken. Zwar lässt sich Castels Hypothese, derzufolge zunehmende Beschäftigungsunsicherheit mit porösen sozialen Netzwerken korreliert, in dieser Pauschalität nicht bestätigen. Aber es gibt doch empirische Belege, die dafür sprechen, dass vor allem sozial Ausgegrenzte zu einer Homogenisierung ihrer sozialen Verkehrskreise tendieren (Marquardsen 2011).

Die Homogenisierung von Sozialbeziehungen lässt sich als Wechselspiel von Fremd- und Selbstdisziplinierung interpretieren. Arbeitslosen fehlen häufig Equipment und finanzielle Mittel, um sich an den Freizeitaktivitäten von Freunden und Bekannten überhaupt beteiligen $\mathrm{zu}$ können. Deshalb meiden sie Alltagssituationen, in denen der prekäre Status zum Problem wird. Umgekehrt verheißen nur solche sozialen Kontakte, Freundschaften und Beziehungen ein Minimum an sozialer Stabilität, in denen gemeinsam geteilte Erfahrungen zur Sprache kommen können, ohne sogleich Stigmatisierungen auszulösen. In solchen Konstellationen erzeugen der Freundeskreis oder gar die eigene Familie informelle Prüfungssituationen. Zur Prüfung steht die Zugangsberechtigung zum Kommunikations- und Lebenszusammenhang. Scham und die Angst, mit einer Welt konfrontiert zu werden, in der man selbst nicht (mehr) leben kann, führen dazu, dass man sich nur noch unter Seinesgleichen bewegt. Dabei gilt, dass die Tendenz zum Selbstausschluss aus sozialen Netzen in prosperierenden Regionen besonders ausgeprägt ist (vgl. Dörre et al. 2009, S. 559ff.).

Bewährungsproben der Lebensführung bewirken, dass das Machtgefälle am Arbeitsmarkt sich im Privaten in Gestalt reduzierter Zeitsouveränität und durch (Selbst-)Ausschluss aus sozialen Netzen gewissermaßen verdoppelt. Verbliebene Sozialkontakte werden von Arbeitslosen und prekär Beschäftigten häufig mit Zähnen und Klauen verteidigt. So scheiterte z.B. ein Pilotversuch, Jugendliche über Leiharbeit in den ersten Arbeitsmarkt zu integrieren, an der Mobilitätserwartung der Leiharbeitsfirma. Als die Jugendlichen erfuhren, dass sie im gesamten Bundesgebiet eingesetzt werden sollten, verweigerten sie die Unterschrift unter den Arbeitsvertrag. Sie fürchteten, Sicherheit stiftende Sozialbeziehungen zugunsten unsicherer Beschäftigung aufgeben zu müssen (Brinkmann et al. 2009).

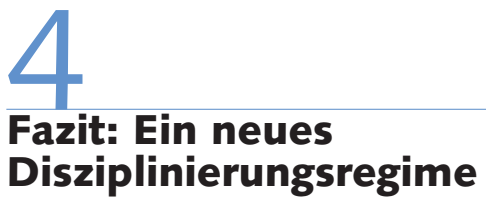

Gemeinsam wirken betriebliche, staatliche und private Bewährungsproben als Treiber des historisch neuen Disziplinarregimes diskriminierender Prekarität. Diese Form der Prekarität korrespondiert mit Machtasymmetrien, die deutlich über das hinausgehen, was in den 1980er Jahren als ,sekundäres Machtgefälle“ am Arbeitsmarkt bezeichnet wurde (Offe/Hinrichs 1984, S. 44ff.). Disziplinierungsmechanismen erfassen nicht mehr „nur“ Problemgruppen, die ihre ,'gebrochene Normalität" als Arbeitnehmer" mittels Ausübung von Alternativrollen zumindest subjektiv entschärfen und so zu überdurchschnittlich geduldigen und belastbaren Arbeitnehmern werden (ebd., S. 79). Diskriminierende Prekarität erzeugt ein Machtgefälle, das mit den diversen Segmenten der Erwerbsarbeitsgesellschaft zugleich die Reproduktionsverhältnisse durchdringt. Sie bringt einen gesellschaftlichen Sonderstatus hervor, der sowohl aus der Perspektive noch gesicherter Gruppen als auch in der Selbstwahrnehmung der Prekarisierten als Minderheitenproblematik erscheint.

In der gesellschaftlichen Wahrnehmung ist eine soziale Hierarchie entstanden, in der diejenigen, die in den schwierigsten Verhältnissen leben müssen und die zugleich über die geringsten Machtressourcen verfügen, sich als Angehörige minoritärer Gruppen erleben. Die alltägliche Lebenspraxis dieser „Minoritäten“" weicht erheblich von den Standards der „Mehrheitsgesellschaft“ ab. Der Sonderstatus wird auch über Genus, Nationalität und
Ethnie konstruiert; er stellt jedoch etwas Eigenes dar. Prekär zu leben bedeutet, bei der Verfügung über Machtressourcen schwach zu sein. Im Erwerbssystem verfügen die Prekarisierten kaum über kollektive, institutionalisierte Organisationsmacht. Im Reproduktionsbereich wird die relative Ohnmacht durch fehlende Zeitsouveränität und selektive Zugänge zu sozialen Netzen zusätzlich verstärkt. Wenngleich die schwachen Positionen der Prekarisierten im Produktions- und Reproduktionssektor jeweils besondere Ursachen besitzen, erzeugen sie einen gleichgerichteten Effekt. Immer scheint es, als lasse sich die nächste Stufe in der sozialen Hierarchie, die ein wenig Mehr an „Normalität“ verspricht, durch eigene Anstrengung erklimmen. Für die Sozialhilfeempfängerin, die niemals längerfristig erwerbstätig war, wird die Familiengründung zur letzten Chance, Anschluss an die gesellschaftliche „Normalität“ zu erreichen. Der Ein-Euro-Jobber empfindet die seltene ABM-Maßnahme als „Glückslos in der Lotterie“. Für die ABM'lerin verheißt die Leiharbeit in der Automobilindustrie eine attraktive Perspektive; der Leiharbeiter wiederum beneidet die befristet Beschäftigte, weil diese immerhin auf Zeit der Stammbelegschaft angehört. Stets entscheidet die Verfügung über Zeitressourcen und soziale Kontakte im Lebenszusammenhang mit darüber, ob man die nächste Sprosse auf der Leiter, die nach oben führt, erreicht.

Was aus der gesellschaftlichen Makroperspektive als Blockierung von Lebenschancen interpretiert werden kann, erscheint in der Mikroperspektive als Bewährungschance für kleine, aber subjektiv eminent bedeutsame Positionsverbesserungen. Mehrheitlich fühlen sich die Prekarisierten dabei als Angehörige von Minderheiten, die selbst alles dafür tun müssen, um Anschluss an das „normale Leben“" zu gewinnen. Festangestellte wiederum fürchten nichts mehr, als in der Hierarchie auf einen solchen Minderheitenstatus zurückzufallen. Auf diese Weise erzeugen die Bewährungsproben diskriminierender Prekarität ein feingliedriges System der (De-)Privilegierung, das auch jene diszipliniert, die nicht oder noch nicht in prekären Verhältnissen leben müssen. Das Wechselspiel von Disziplinierung und Selbstdisziplinierung, das den gesamten Lebenszusammenhang durchdringt, maskiert indessen den Herrschaftscharakter von Prekarisierungsprozessen. Das skiz- 
zierte Disziplinarregime ist Bestandteil eines Kontrollmodus, der die eigentlich Herrschenden weitgehend unsichtbar macht. In ihrem Bemühen, die Bewährungsproben diskriminierender Prekarität erfolgreich zu absolvieren oder sie nach Möglichkeit zu vermeiden, geraten die eigentlichen „Verantwortungsträger“, die die Prüfungsformate festlegen, aus dem Blick. Stattdessen nimmt auch unter den Subalternen die Neigung zu exklusiven, ausschließenden Gruppensolidaritäten zu.

\section{Intervention durch Sozialkritik}

Ändern lässt sich dies nur, wenn es gelingt, die Prüfungsformate samt der mit ihnen verwobenen Rechtfertigungsordnungen durch eine erneuerte Sozialkritik nachhaltig zu beeinflussen. Gegenwärtig stoßen drei Formen der Prekarisierungskritik auf gesellschaftliche Resonanz. Die immanente Kritik greift die alltägliche Perspektive eines (Über-)Lebens in prekären Verhältnissen auf und drängt dazu, „nach dem kreativen Umgang der Subjekte mit gesellschaftlichen Verunsicherungsprozessen" zu forschen (Manske/Pühl 2010, S. 10). Obwohl diese Kritikvariante z. B. mit den neuen Dienstbotenverhältnissen in zahlungskräftigen Haushalten zugleich den
Herrschaftscharakter diskriminierender Prekarität im Reproduktionssektor aufdeckt, fällt es ihr schwer, Übergänge vom individuellen zu kollektivem Handeln zu bestimmen. Es geht ihr vor allem um eine gerechte Verteilung von Ressourcen, die eine erfolgreiche Bewältigung von Bewährungsproben ermöglichen. Die pragmatische Kritik zielt hingegen auf die - kollektive - Eindämmung von Prekarität. Mit gesetzlichen Mindestlöhnen und EqualPay-Regelungen beabsichtigt sie, uneindeutige in legitime Bewährungsproben zu verwandeln. Weil sie im Reproduktionssektor, aber auch in Branchen mit hohen Frauenanteilen wie dem Pflege- und Gesundheitssektor, kaum verankert ist, muss sie häufig an die „Starken“ appellieren, um den „schwachen“, prekarisierten Gruppen zu helfen. Unklar bleibt bislang, ob diese Variante der Sozialkritik überhaupt zu einer Sprache findet, die es ihr erlaubt, die Schranken einer lediglich kompetitiven Solidarität noch halbwegs geschützter Beschäftigtengruppen zu überschreiten.

Von beiden Ausprägungen unterscheidet sich eine radikale Sozialkritik, deren Programm bislang nur in Andeutungen vorliegt. Luc Boltanski schlägt vor, die Großgruppen der prekarisierten „Verantwortungslosen“, ihre Bewegungen und Revolten, die häufig nihilistisch erscheinen und die „Form unüberlegten Ausrastens“ annehmen (Boltanski 2010, S. 226ff.), als Träger alltäglicher Sozialkritik ernst zu nehmen. Der antiinstitutionalistische Gestus, mit dem diese Kritikvariante operiert, mag Gewerkschafter befremden. Eine diskursive Leistung dieser Kritikvariante besteht jedoch darin, dass sie den vermeintlichen Minderheitenstatus prekarisierter Gruppen attackiert und damit zugleich die einflussreichen Minoritäten herrschender „Verantwortungsträger" sichtbar macht. Schon ein Blick auf transnationale Wertschöpfungsketten genügt, um die Relevanz einer Sozialkritik anzuerkennen, die Prekarisierung und Informalität in der globalen Dimension als Mehrheitsproblematik begreift (Jütting/Laiglesia 2009; Roth 2010, S. 119ff.).

Jede der genannten Kritikvarianten besitzt ihre eigene Legitimität. Gegenwärtig gelingt es jedoch den potenziellen Trägern weder praktisch-politisch noch wissenschaftlich, die unterschiedlichen Kritikperspektiven bei Wahrung ihrer Eigenständigkeit zu einer produktiven Synthese zusammenzufügen. Erst ein realistisches Bewusstsein der transnationalen Vielfalt unsicherer Arbeits- und Lebensverhältnisse wie auch der Pluralität widerständiger Bewegungen, bietet die Chance, den Herrschaftsmodus diskriminierender Prekarität als solchen infrage zu stellen. Auch deshalb ist es für eine sozialwissenschaftlich fundierte Sozialkritik zwingend nötig, keine der genannten Kritikperspektiven vorschnell aus ihren Überlegungen auszublenden.

\section{LITERATUR}

Balibar, E./Wallerstein, I. (1988): Race, Nation, Classe. Les identés ambiugés, Paris

Behr, M. (2009): Arbeitsbewusstsein und Interessenorientierung bei $X$. Auswertung und Analyse einer Belegschaftsbefragung bei den Beschäftigten der X.-Betriebe 2008, Otto-Brenner-Stiftung, Frankfurt a. M. Bescherer, P./Röbenack, S./Schierhorn, K. (2008): „Nach Hartz IV: Erwerbsorientierungen von Arbeitslosen“, in: Aus Politik und Zeitgeschichte (33-34), S. 19-24

Boltanski, L. (2010): Soziologie und Sozialkritik, Frankfurter AdornoVorlesungen 2008, Berlin

Boltanski, L./Chiapello, È. (2003): Der neue Geist des Kapitalismus, Konstanz
Booth, M./Dörre, K./Haubner, T./Marquardsen, K./Scherschel, K./ Schierhorn, K. (2011): Bewährungsproben für die Unterschicht. Soziale Wirkungen strenger Zumutbarkeit, Diskussionspapier für den SFB 580, hrsg. von der Friedrich-Schiller-Universität Jena

Booth, M./Scherschel, K./Schierhorn, K. (2010): Aktivierende Arbeitsmarktpolitik in Deutschland - zentrale Entwicklungslinien und empirische Befunde, Diskussionspapier des B9-Projekts im SFB 580, hrsg. von der Friedrich-Schiller-Universität Jena

Bourdieu, P./Balazs, G./Beaud, S./Broccolichi, S./Champagne, P./ Christin, R./Lenoir, R./Oeuvrard, F./Pialoux, M./Sayad, A./ Schultheis, F./Soulié, C. (1997): Das Elend der Welt. Zeugnisse und Diagnosen alltäglichen Leidens, Konstanz 
Brinkmann, U./Dörre, K./Röbenack, S. gem. m. Kraemer, K./ Speidel, F. (2006): Prekäre Arbeit. Ursachen, Ausmaß, soziale Folgen und subjektive Verarbeitungsformen unsicherer Beschäftigungsverhältnisse. Eine Expertise im Auftrag der Friedrich-Ebert-Stiftung, Bonn Brinkmann, U./Eversberg, D./Scherschel, K. (2009): Endbericht Evaluation „ZACQ". Qualitativer Teil: Implementationsanalysen, Wirkungsanalysen, Forschungsbericht an das Institut für Arbeitsmarktund Berufsforschung, hrsg. von der Friedrich-Schiller-Universität Jena Castel, R. (2000): Die Metamorphosen der sozialen Frage. Eine Chronik der Lohnarbeit, Konstanz

Castel, R. (2005): Die Stärkung des Sozialen. Leben im neuen Wohlfahrtsstaat, Hamburg

Crozier, M./Friedberg, E. (1993): Die Zwänge kollektiven Handelns. Über Macht und Organisation, Frankfurt a. M.

Dörre, K. (2009): Prekarität im Finanzmarkt-Kapitalismus,

in: Castel, R./Dörre, K. (Hrsg.): Prekarität, Abstieg, Ausgrenzung. Die soziale Frage am Beginn des 21. Jahrhunderts, Frankfurt a. M./

New York, S. 35-64

Dörre, K./Behr, M./Eversberg, D./Schierhorn, K. (2009): Krise ohne Krisenbewusstsein? Zur subjektiven Dimension kapitalistischer Landnahmen, in: PROKLA 157, 39 (4), S. 559-576

Dörre, K./Hänel, A./Holst, H./Matuschek, I. (2011): Guter Betrieb, schlechte Gesellschaft? Arbeits- und Gesellschaftsbewusstsein im Prozess kapitalistischer Landnahme, in: Koppetsch, C. (Hrsg.): Nachrichten aus den Innenwelten das Kapitalismus, Wiesbaden (im Erscheinen)

Hohendanner, C. (2010): Unsichere Zeiten, unsichere Verträge? Befristete Arbeitsverträge zwischen Auf- und Abschwung, IAB-Kurzbericht 14, hrsg. vom Institut für Arbeitsmarkt- und Berufsforschung, Nürnberg
Holst, H./Matuschek, I. (2011): Sicher durch die Krise? Leiharbeit, Krise und Interessenvertretung, in: Haipeter, T./Dörre, K. (Hrsg.): Modernisierung von Gewerkschaften, Wiesbaden (im Erscheinen) Holst, H./Nachtwey, O./Dörre, K. (2009): Funktionswandel von Leiharbeit. Neue Nutzungsstrategien und ihre arbeits- und mitbestimmungspolitischen Folgen, OBS-Arbeitsheft (61), hrsg. von der Otto-Brenner-Stiftung, Berlin

Holst, H./Nachtwey, O./Dörre, K. (2010): The Strategic Use of Temporary Agency Work - Functional Change of a Non-standard Form of Employment, in: International Journal of Action Research 6 (1), S. $108-138$

Jütting, P.J./Laiglesia, J.R. (2009): Is Informal normal? Towards more and better Jobs in Developing Countries, Paris

Manske, A./Pühl, K. (2010): Einleitung, in: Manske, A./Pöhl, K. (Hrsg.): Prekarisierung zwischen Anomie und Normalisierung. Geschlechtertheoretische Bestimmungen, Münster

Marquardsen, K. (2011): Aktivierung und soziale Netzwerke. Die Dynamik sozialer Beziehungen unter dem Druck der Erwerbslosigkeit. Dissertation zur Erlangung des Grades doctor philosophiae, Jena Negt, O. (1984): Lebendige Arbeit, enteignete Zeit. Politische und kulturelle Dimensionen des Kampfes um die Arbeitszeit, Frankfurt a. M./ New York

Offe, C./Hinrichs, K. (1984): Sozialökonomie des Arbeitsmarktes: primäres und sekundäres Machtgefälle, in: Offe, C. (Hrsg.): "Arbeitsgesellschaft". Strukturprobleme und Zukunftsperspektiven, Frankfurt a. M./New York, S. 44-86

Pelizzari, A. (2009): Dynamiken der Prekarisierung. Atypische Erwerbsverhältnisse und milieuspezifische Unsicherheitsbewältigung, Konstanz Roth, K.-H. (2010): Die globale Krise. Globale Krise - Globale Proletarisierung - Gegenperspektiven, Hamburg 